\title{
Placental Site Plaque of the Uterine Cervix Misinterpreted as Low-Grade Squamous Intraepithelial Lesion in Liquid-Based Cervicovaginal Cytology: Usefulness of Inhibin- $\alpha$ Immunocytochemistry
}

\author{
Hyunjin Kim ${ }^{a}$ Ha Young Woo ${ }^{b}$ Sang Hwa Lee ${ }^{c}$ Sung-Im Do ${ }^{d}$ \\ Hyun-Soo Kim ${ }^{a}$ \\ aDepartment of Pathology and Translational Genomics, Samsung Medical Center, \\ Sungkyunkwan University School of Medicine, Seoul, Republic of Korea; ${ }^{b}$ Department of \\ Pathology, Severance Hospital, Yonsei University College of Medicine, Seoul, Republic \\ of Korea; 'Pathology Center, Seegene Medical Foundation, Seoul, Republic of Korea; \\ ${ }^{\mathrm{d} D e p a r t m e n t ~ o f ~ P a t h o l o g y, ~ K a n g b u k ~ S a m s u n g ~ H o s p i t a l, ~ S u n g k y u n k w a n ~ U n i v e r s i t y ~ S c h o o l ~}$ \\ of Medicine, Seoul, Republic of Korea
}

\section{Keywords}

Cervix - Liquid-based cytology · Low-grade squamous intraepithelial lesion · Placental site plaque

\begin{abstract}
Cytological features of placental site plaques in liquid-based cervicovaginal preparations have been seldom documented in the literature. We present a rare case of endocervical placental site plaque misinterpreted as a low-grade squamous intraepithelial lesion in a liquid-based cytological preparation. A 32-year-old woman with polycystic ovarian syndrome gave birth 7 months previously. After delivery, she was diagnosed with cervical low-grade squamous intraepithelial lesion during routine cytological examination. Cytologically, many atypical cells showed large hyperchromatic nuclei with irregular membranes. The perinuclear cytoplasmic clearing closely resembled koilocytosis. Histologically, the endocervix showed typical histological features of a placental site plaque. Immunohistochemically, the trophoblasts were positive for $p 63, C D 10$, and inhibin- $\alpha$ but negative for $p 16$. Based on genotyping, both the cytological and biopsied specimens tested negative for human papillomavirus. We re-examined the liquid-based preparation cytology slides thoroughly and concluded that the atypical cells initially misinterpreted as low-grade squamous intraepithelial lesion were actually trophoblasts. Immunocytochemical staining revealed uniform cytoplasmic inhibin- $\alpha$ expression in the trophoblasts. In summary, we demonstrated that endocervical placental site plaques
\end{abstract}


can mimic low-grade squamous intraepithelial lesions in liquid-based cytological preparations. Immunocytochemical staining results and negative results on human papillomavirus genotyping further support that atypical cells resembling koilocytes are trophoblasts obtained from the placental site plaque.

(c) 2020 The Author(s).

Published by S. Karger AG, Basel

\section{Introduction}

Liquid-based cytological features of placental site plaque have been seldom documented in the literature. We recently experienced an extremely rare case of placental site plaque occurring in the endocervix, cytologically mimicking a low-grade squamous intraepithelial lesion in liquid-based cytological preparation. We describe the liquid-based cytomorphology of the placental site plaque resembling that of low-grade squamous intraepithelial lesion and its immunocytochemical staining results. Our report will improve the understanding of this rare condition and offer insight to pathologists faced with diagnostic decisions.

\section{Case Presentation}

A 32-year-old woman who gave birth 7 months previously was diagnosed with a cervical low-grade squamous intraepithelial lesion during cytological examination. Liquid-based cervicovaginal cytological preparation (Fig. 1) revealed atypical squamous cells with enlarged hyperchromatic nuclei and irregular membranes and occasional intranuclear grooves. The atypical cells showed mild-to-moderate nuclear pleomorphism and perinuclear cytoplasmic clearing. Dense peripheral cytoplasmic condensation, a characteristic of koilocytosis observed in low-grade squamous intraepithelial lesions, was readily identifiable. The diameter of the enlarged nuclei was approximately 3 times that of the adjacent bland-appearing round nuclei. Severe nuclear pleomorphism, mitotic activity, and atypical mitotic figure were absent. Taken together, the cytological diagnosis of low-grade squamous intraepithelial lesion was established $[1,2]$.

Colposcopy-directed cervical punch biopsy and endocervical curettage were performed. The biopsied specimens were fixed in 10\% neutral-buffered formalin and embedded in paraffin blocks. From each formalin-fixed, paraffin-embedded block, 4- $\mu \mathrm{m}$ sections were cut and stained with hematoxylin and eosin. The most representative hematoxylin and eosinstained slides, containing an appropriate volume of lesional tissue, were chosen for immunostaining. The punch biopsy specimen (Fig. 2) revealed that the lesion consisted of relatively well-circumscribed plaques with hyalinized extracellular matrix, in which intermediate trophoblasts were distributed as single cells or clusters. Small-to-medium-sized intermediate trophoblasts were embedded in the hyalinized stroma and exhibited enlarged hyperchromatic nuclei with mild pleomorphism. No severe nuclear pleomorphism or mitotic activity was observed.

Immunohistochemical staining was performed using a compact polymer method (Bond Polymer Refine Detection kit, Leica Biosystems, Newcastle, UK). Four-micrometer formalinfixed, paraffin-embedded sections were incubated with primary antibodies against cytokeratin, p63, inhibin- $\alpha$, and p16. Immunohistochemical staining revealed that the trophoblastic cells were positive for cytokeratin, p63, and inhibin- $\alpha$ but negative for $\mathrm{p} 16$. Both the cytological and biopsied specimens tested negative for human papillomavirus. Based on the history of recent delivery, these histological features were compatible with placental site plaque of the endocervix [3-6].

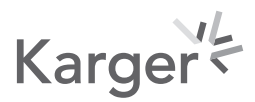




\section{Case Reports in Oncology}

\begin{tabular}{l|l}
\hline Case Rep Oncol 2020;13:1415-1420 \\
\hline DOI: 10.1159/000510310 & $\begin{array}{l}\text { @ 2020 The Author(s). Published by S. Karger AG, Basel } \\
\text { www.karger.com/cro }\end{array}$ \\
\hline
\end{tabular}

Kim et al.: Placental Site Plaque Misinterpreted as Low-Grade Squamous Intraepithelial Lesion

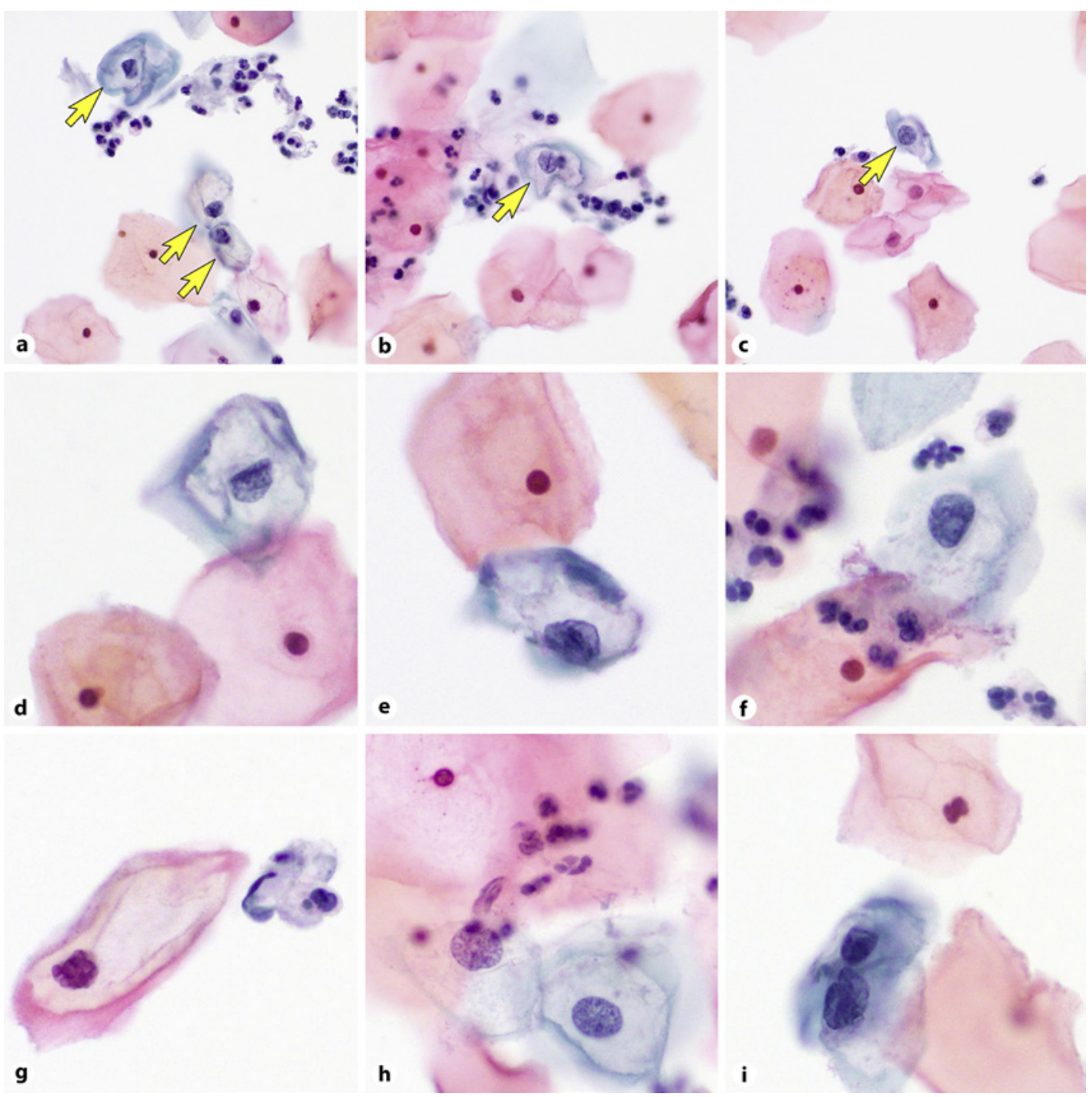

Fig. 1. Cytological features in the liquid-based cervicovaginal preparation initially interpreted as low-grade squamous intraepithelial lesion. a-c The yellow arrows indicate atypical squamous cells with enlarged hyperchromatic nuclei and irregular membranes. These cells are cytomorphologically compatible with lowgrade squamous intraepithelial lesions. Atypical cells show perinuclear cytoplasmic clearing. Dense peripheral cytoplasmic condensation, a characteristic of koilocytosis observed in low-grade squamous intraepithelial lesions, is readily identifiable. Note the intranuclear grooves. $\mathbf{d}-\mathbf{i}$ Higher magnifications reveal enlarged nuclei, of which the diameter is approximately 3 times that of the adjacent bland-appearing round nuclei. The cells show hyperchromasia, membrane irregularity, and mild-to-moderate pleomorphism. Severe pleomorphism, mitotic activity, and atypical mitotic figure are absent. Taken together, the cytological diagnosis of a low-grade squamous intraepithelial lesion is established. Staining method: a-i, Papanicolaou staining. Magnification: a-c, $\times 400 ; \mathbf{d}-\mathbf{i}, \times 1,000$.

We thoroughly reviewed the cytology slide and performed cytohistological correlation (Fig. 3). The trophoblasts observed in the placental site plaque are morphologically concordant with the koilocyte-like atypical cells identified in the liquid-based cytological preparation. Immunocytochemical staining highlighted the trophoblasts that expressed cytoplasmic inhibin- $\alpha$. 


\section{Case Reports in Oncology}

Kim et al.: Placental Site Plaque Misinterpreted as Low-Grade Squamous Intraepithelial Lesion

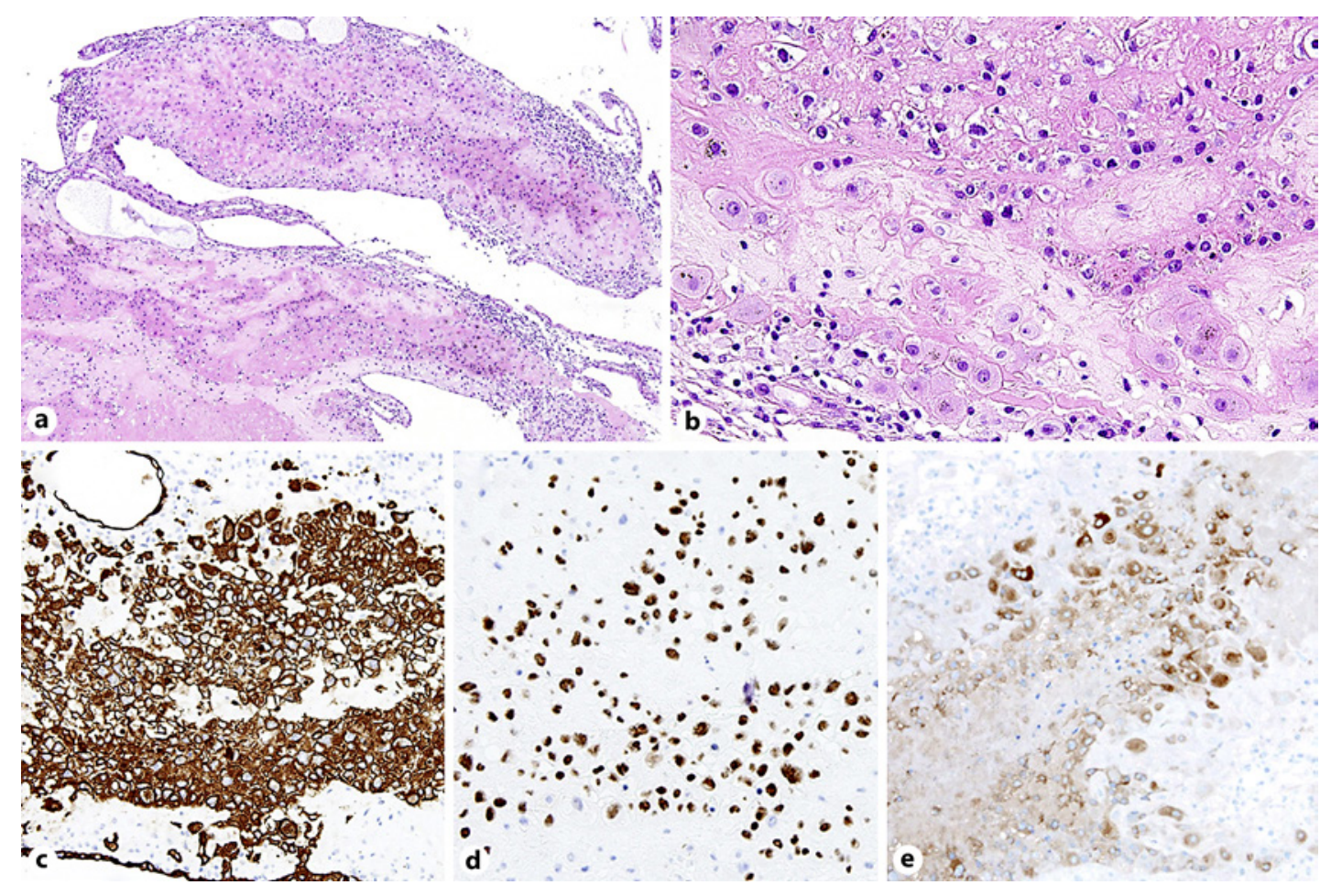

Fig. 2. Histological features and immunohistochemical staining results of the placental site plaques. a The lesion consists of plaques relatively well-circumscribed with hyalinized extracellular matrix, in which intermediate trophoblasts are distributed as single cells or clusters. b Small-to-medium-sized intermediate trophoblasts are embedded in the hyalinized stroma and exhibit enlarged hyperchromatic nuclei with mild pleomorphism. Decidualized stromal cells are polygonal and possess abundant eosinophilic cytoplasm. No mitotic activity is observed in the trophoblasts or decidualized cells. Immunohistochemical staining reveals that the trophoblastic cells are positive for cytokeratin (membranous staining) (c), p63 (nuclear staining) (d), and inhibin- $\alpha$ (cytoplasmic staining) (e). Staining method: a, b, hematoxylin and eosin staining; c-e, polymer method. Magnification: $\mathbf{a}, \times 100$; b-e, $\times 200$.

\section{Discussion}

We found that intermediate trophoblasts of placental site plaques closely mimic lowgrade squamous intraepithelial lesion in liquid-based cytological preparation. They showed nuclear enlargement, hyperchromasia, membrane irregularity, and perinuclear halos with peripheral condensation, resembling koilocytes. Trophoblasts may be mistaken for atypical squamous cells on liquid-based cytological preparation [7]. In fact, we cannot completely exclude the possible presence of cervical low-grade squamous intraepithelial lesions in other portions of the cervix that were not sampled during punch biopsy or endocervical curettage. Nevertheless, a history of recent delivery, inhibin- $\alpha$ immunoreactivity [8-11], cytohistological concordance, and lack of human papillomavirus infection strongly suggest that koilocyte-like cells are not squamous epithelial cells but trophoblasts obtained from placental site plaques. 


\section{Case Reports in Oncology}

\begin{tabular}{l|l}
\hline Case Rep Oncol 2020;13:1415-1420 \\
\hline DOI: 10.1159/000510310 & $\begin{array}{l}\text { @ 2020 The Author(s). Published by S. Karger AG, Basel } \\
\text { www.karger.com/cro }\end{array}$ \\
\hline
\end{tabular}

Kim et al.: Placental Site Plaque Misinterpreted as Low-Grade Squamous Intraepithelial Lesion

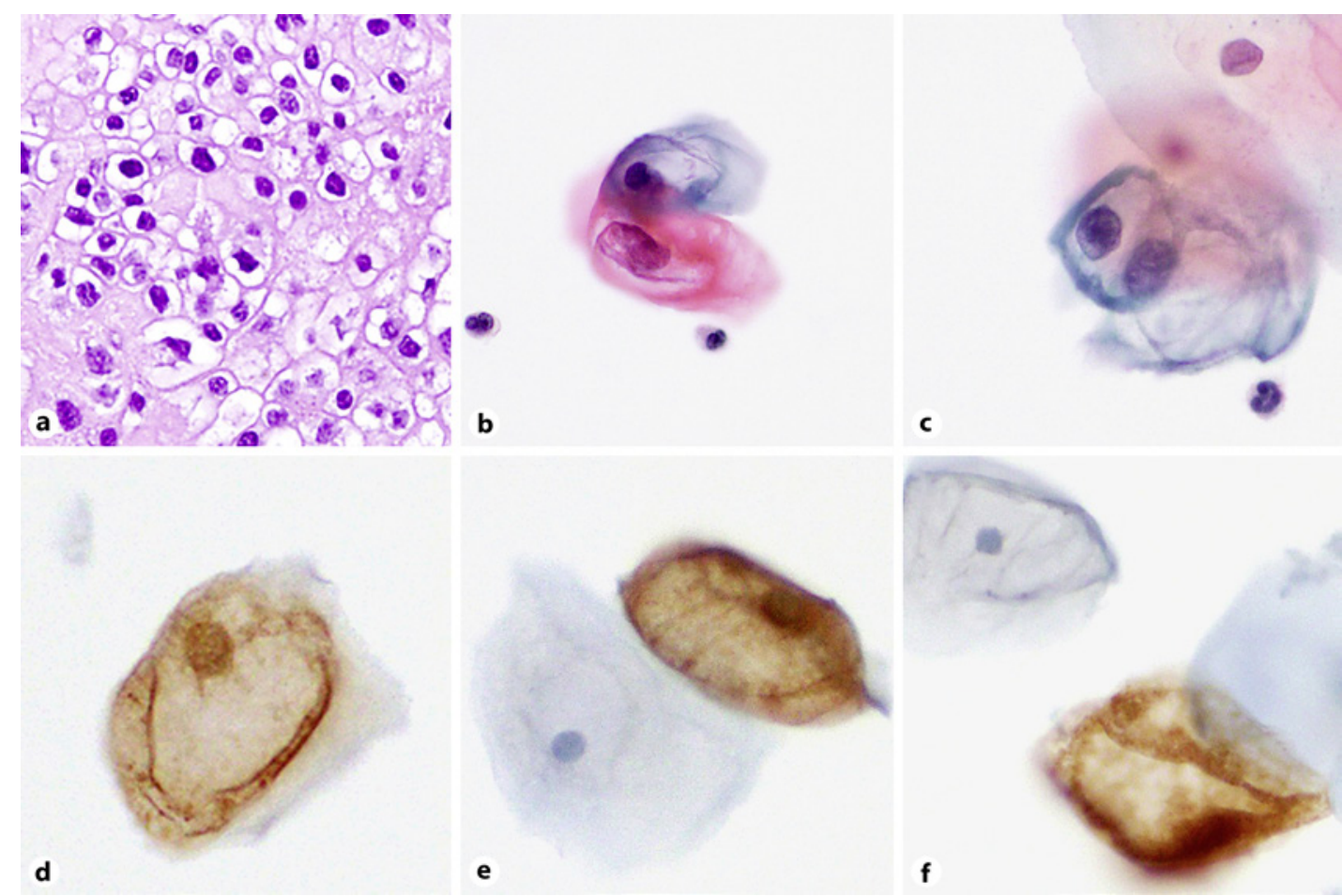

Fig. 3. Re-examined cytological features and immunocytochemical staining results of the liquid-based cervicovaginal preparation. We thoroughly reviewed the cytology slide and performed cytohistological correlation. a-c The trophoblasts observed in the placental site plaque (a) are morphologically concordant with the koilocyte-like atypical cells identified in the liquid-based cytological preparation (b-d). d-f Immunocytochemical staining highlights the trophoblasts that express cytoplasmic inhibin- $\alpha$. Staining method: a, hematoxylin and eosin staining; b, c, Papanicolaou staining; d-f, polymer method. Magnification: a, $\times 400$; b-f, $\times 1,000$.

\section{Statement of Ethics}

A written informed consent for publication was obtained from the patient. This study (2017-3062-001) was reviewed and approved by the Institutional Review Board of Severance Hospital (Seoul, Republic of Korea).

\section{Conflict of Interest Statement}

The authors have no conflicts of interest to declare.

\section{Funding Sources}

This research was supported by the National Research Foundation of Korea (NRF) grant funded by the Korean government (Ministry of Science and ICT) (2018R1C1B5043725). 


\section{Case Reports in Oncology}

\begin{tabular}{l|l}
\hline Case Rep Oncol 2020;13:1415-1420 \\
\hline DOI: 10.1159/000510310 & $\begin{array}{l}\text { @ 2020 The Author(s). Published by S. Karger AG, Basel } \\
\text { www.karger.com/cro }\end{array}$ \\
\hline
\end{tabular}

Kim et al.: Placental Site Plaque Misinterpreted as Low-Grade Squamous Intraepithelial Lesion

\section{Author Contributions}

Hyunjin Kim: conceptualization, data collection, data analysis, manuscript drafting, and project administration; Ha Young Woo: data collection and manuscript editing; Sang Hwa Lee: manuscript editing; Sung-Im Do: conceptualization, funding acquisition, manuscript editing, and supervision; Hyun-Soo Kim: conceptualization, data collection, data analysis, manuscript drafting, manuscript editing, and supervision. All authors read and approved the final manuscript.

\section{References}

1 Nayar R, Wilbur DC. The Bethesda System for Reporting Cervical Cytology: Definitions, Criteria, and Explanatory Notes. Springer; 2015.

2 Goldblum JR, Lamps LW, McKenney JK, Myers JL. Rosai and Ackerman's Surgical Pathology. Elsevier; 2017.

3 Young RH, Kurman RJ, Scully RE. Placental site nodules and plaques. A clinicopathologic analysis of 20 cases. Am J Surg Pathol. 1990 Nov;14(11):1001-9.

4 Santos LD, Fernando SS, Yong JL, Killingsworth MC, Wu XJ, Kennerson AR. Placental site nodules and plaques: a clinicopathological and immunohistochemical study of 25 cases with ultrastructural findings. Pathology. 1999 Nov;31(4):328-36.

5 Shih IM, Kurman RJ. The pathology of intermediate trophoblastic tumors and tumor-like lesions. Int J Gynecol Pathol. 2001 Jan;20(1):31-47.

6 Kurman RJ, Carcangiu ML, Herrington CS, Young RH. WHO classification of tumours of female reproductive organs. IARC; 2014.

7 Hakima L, Kaplan RE, Guo M, Hoda RS. Decidual cells may be mistaken for glandular or squamous atypia on ThinPrep Pap test. Diagn Cytopathol. 2013 Oct;41(10):886-8.

8 Shitabata PK, Rutgers JL. The placental site nodule: an immunohistochemical study. Hum Pathol. 1994 Dec; 25(12):1295-301.

9 Shih IM, Kurman RJ. Immunohistochemical localization of inhibin-alpha in the placenta and gestational trophoblastic lesions. Int J Gynecol Pathol. 1999 Apr;18(2):144-50.

10 Houghton 0, McCluggage WG. The expression and diagnostic utility of p63 in the female genital tract. Adv Anat Pathol. 2009 Sep;16(5):316-21.

11 Kondi-Pafiti A, Grigoriadis C, Samiotaki D, Filippidou-Giannopoulou A, Kleanthis C, Hassiakos D. Immunohistochemical study of inhibin A and B expression in placentas from normal and pathological gestations. Clin Exp Obstet Gynecol. 2013;40(1):109-12. 